\title{
INVARIANT DIFFERENTIAL EQUATIONS ON CERTAIN SEMISIMPLE LIE GROUPS
}

BY

F. ROUVIÈRE

\begin{abstract}
If $G$ is a semisimple Lie group with one conjugacy class of Cartan subalgebras (e.g. a complex semisimple Lie group), a bi-invariant differential equation on $G$ can be reduced by means of the Radon transform to one on the subgroup $M A$. In particular, all polynomials of the Casimir operator have a central fundamental solution, and are solvable in $C^{\infty}(G)$; but, for $G$ complex, the "imaginary" Casimir operator is not.
\end{abstract}

0. Introduction. In this paper we study global solvability of a bi-invariant differential operator $P$ on a noncompact semisimple Lie group $G$ with finite center. It is known that $P$ is locally solvable, i.e. every point in $G$ has a neighborhood $V$ such that $P C^{\infty}(V) \supset \mathscr{D}(V)$, where $\mathscr{D}$ means $C^{\infty}$ with compact support (Helgason [11, p. 477]); also, globally but modulo a maximal compact subgroup $K$, there are fundamental solution and solvability in $C^{\infty}$ on the symmetric space $G / K$, if $P$ does not induce 0 on it (Helgason [8, p. 578]; [11, p. 473]).

Globally on $G$ itself, difficulties may arise from the compact subgroup $K$, more precisely from its subgroup $M$. We restrict ourselves here to the case where $G$ has but one conjugacy class of Cartan subalgebras (e.g. a complex semisimple Lie group). Then, owing to the absence of discrete series in the Plancherel formula, we can reduce the problem for $P$ on $G$ to a similar problem for a differential operator $\tau(P)$ on the group $M A$ (a Cartan subgroup, for $G$ complex). Our main result is

4.2. THEOREM. If $\tau(P)$ has a fundamental solution on $M A$, then $P$ has one on $G$, which is central, and $P C^{\infty}(G)=C^{\infty}(G)$.

An explicit criterion is given for $\tau(P)$ to be solvable on $M A$, which is the direct product of compact and abelian Lie groups. This applies in particular to all polynomials of the Casimir operator (5.1. Corollary). On the other hand, a simple necessary condition is given (4.1. Proposition) and we show that the "imaginary" Casimir operator of a complex semisimple Lie group is not

Received by the editors June 7, 1977.

AMS (MOS) subject classifications (1970). Primary 58G99; Secondary $22 \mathrm{E} 30$.

Key words and phrases. Semisimple Lie groups, bi-invariant differential operators, Radon transform, Casimir operator.

- American Mathematical Society 1978 
globally solvable (5.3. Proposition); more precisely, polynomials of the Casimir and "imaginary" Casimir are solvable on $G$ if and only if they do not induce 0 on $G / K$, when split rank of $G$ is $>2$ (5.4. Proposition), but this equivalence is no longer true for more general bi-invariant operators.

Notations are defined in $\$ 1$. In $\$ 2$ we introduce an integral transform on $G$ generalizing the Radon transform on $G / K$, which gives a convenient framework to state Johnson's (or Želobenko's) Paley-Wiener theorem; $\tau(P)$ appears when studying the action of $P$ on this transform; most results in this section are not new, at least when $G$ is complex and $M A$ a Cartan subgroup. $\$ 3$ is devoted to a study of $P$-convexity and existence of fundamental solutions on $M A$ by standard Fourier analysis on compact and abelian groups. In $\$ 4$ are proved our main results. In $\$ 5$, finally, examples and couterexamples are given, considering polynomials of the Casimir and "imaginary" Casimir operator.

Part of the results of this paper, for $G$ complex, were announced in A. Cerezo and F. Rouvière, Séminaire Goulaouic-Schwartz 1972-1973, exposé $\mathrm{n}^{\circ} 10$ (École Polytechnique, Paris).

1. Notation. (a) General notation. As usual $\mathbf{R}, \mathbf{C}, \mathrm{Z}, \mathbf{N}$ denote the sets of real numbers, complex numbers, integers, and nonnegative integers, respectively.

All Lie groups will be real. If $G$ is a Lie group, we denote by $1, g$, exp, Ad, ad, the identity element, Lie algebra, exponential mapping, adjoint representation of $G$, and $g$, respectively. Let $\mathscr{D}(G)$ be the space of complex-valued $C^{\infty}$ functions with compact support on $G, \Phi^{\prime}(G)$ the dual space, $\mathcal{E}^{\prime}(G)$ the subspace of distributions with compact support, $\delta_{G}$ the Dirac measure of $G$ at 1 ; supp means support.

Let $U(\mathrm{~g})$ be the complexified universal enveloping algebra of the real Lie algebra $g, Z(g)$ its center; in particular, when $g$ is a complex Lie algebra, $U(g)$ is the enveloping algebra of the complexification of the underlying real Lie algebra. $U(\mathrm{~g})$, resp. $Z(\mathrm{~g})$, will be identified with the algebra $D(G)$, resp. $Z(G)$, of left invariant, resp. bi-invariant, differential operators with complex coefficients on $G$. The transpose $P^{*}$ of $P \in D(G)$ is defined by

$$
\int_{G} P^{*} f(x) g(x) d x=\int_{G} f(x) P g(x) d x,
$$

$f, g \in \mathscr{D}(G)$, where $d x$ is a right-invariant Haar measure on $G$. Since $X^{*}=-X$ for $X \in \mathrm{g}$ it is clear that, if $H$ is a Lie subgroup of $G$ and $P \in D(H) \subset D(G)$, the transposes of $P$ defined according to $G$ and $H$ are the same; $Z(G)$ is stable under transpose.

For $f \in \mathscr{D}(G), P \in D(G)$, we define $f^{\prime}$ and $P^{\prime}$ by $f^{\prime}(x)=f\left(x^{-1}\right),(P f)^{\prime}=$ $P^{\prime} f^{\prime}$. The following lemma will be used several times.

1.1. Lemax. If $G$ is unimodular and $P \in Z(G)$, then $P^{*}=P^{\prime}$. 
Proof. By unimodularity

$$
\int_{G} f(x y) g\left(x^{-1}\right) d x=\int_{G} f\left(x^{-1}\right) g(y x) d x
$$

for $f, g \in \mathscr{D}(G), y \in G$; therefore applying $P$ on the $y$-variable

$$
\int\left(P_{y} f(x y)\right)_{y=1} g\left(x^{-1}\right) d x=\int f\left(x^{-1}\right)\left(P_{y} g(y x)\right)_{y=1} d x .
$$

In view of left and right invariance of $P$, this yields

$$
\int P f(x) g^{\prime}(x) d x=\int f^{\prime}(x) P g(x) d x
$$

or

$$
\int f(x) P^{*} g^{\prime}(x) d x=\int f(x)(P g)^{\prime}(x) d x,
$$

which proves the lemma.

(b) Semisimple notation. Throughout the paper $G$ will denote a connected noncompact linear semisimple Lie group. Let $B$ be the Killing form of $g, \theta$ a Cartan involution, $\mathfrak{g}=\mathfrak{f}+\mathfrak{p}$ the corresponding decomposition of $\mathfrak{g}$ into eigenspaces of $\theta, \mathfrak{a}$ a maximal abelian subspace of $\mathfrak{p}, \mathfrak{a}^{*}$ the dual of $\mathfrak{a}, \mathfrak{a}_{c}^{*}$ the complexification of $a^{*}$, and $\mathfrak{m}$ the centralizer of $a$ in $\mathfrak{t}$. Let $\mathfrak{n}$, resp. $\overline{\mathfrak{n}}=\theta \mathfrak{n}$, denote the sum of root spaces

$$
\mathfrak{g}_{\alpha}=\{X \in \mathfrak{g} \mid[H, X]=\alpha(H) X \text { for all } H \in \mathfrak{a}\}
$$

corresponding to positive, resp. negative, roots $\alpha$, for some ordering on the set of roots. Then $\mathfrak{g}=\mathfrak{h}+\mathfrak{n}+\overline{\mathfrak{n}}$ (direct sum), setting $\mathfrak{h}=\mathfrak{m}+\mathfrak{a}$ (observe that $\mathfrak{h}$ is a Cartan subalgebra of $g$ when $g$ admits a complex structure); also $\mathfrak{g}=\mathfrak{f}+\mathfrak{a}+\mathfrak{n}$ (direct sum). Let $\rho$ be the half sum of positive roots, counted with their multiplicities.

Let $K, A, N$ be the Lie subgroups of $G$ with Lie algebras $\mathfrak{f}, \mathfrak{a}, \mathfrak{n}$, and let $M$ (resp. $M^{\prime}$ ) denote the centralizer (resp. normalizer) of $A$ in $K, W=M^{\prime} / M$ the Weyl group. $K, M, M^{\prime}$ are compact, $A$ abelian, $N$ nilpotent, and $W$ is finite; then $G=K A N$ (Iwasawa decomposition), and $G=K A K$ (Cartan decomposition; here the $K$-components are not unique, and the $A$-component is unique modulo $W$ ). We set $H=M A$, a direct product. For $a \in A$ and $\nu \in a_{c}^{*}, a^{\nu}$ will mean $e^{\nu(\log a)}$, where $\log$ is the inverse map of exp: $a \rightarrow A . d g$, $d k, d a, d n, d m$ will denote Haar measures on $G, K, A, N, M$, respectively, normalized so that $\int_{K} d k=\int_{M} d m=1$, and for $f \in \mathscr{Q}(G)$,

$$
\int_{G} f(g) d g=\int_{K \times A \times N} f(k a n) a^{2 \rho} d k d a d n=\int_{K \times N \times A} f(k n a) d k d n d a .
$$

We consider two "norms" on $G$, both extending the natural norm $|a|=$ $(B(\log a, \log a))^{1 / 2}$ on $A$; for $g \in G$, we set $|g|=|a|$ if $g=k_{1} a k_{2}$ is any 
Cartan decomposition of $g$, and $\|g\|=\left|a^{\prime}\right|$ if $g=k^{\prime} a^{\prime} n^{\prime}$ is the Iwasawa decomposition $\left(k_{1}, k_{2}, k^{\prime} \in K, a, a^{\prime} \in A, n^{\prime} \in N\right)$.

Finally for $\xi \in \hat{M}$, the set of (equivalence classes of) irreducible unitary representations of $M$, and $\nu \in \mathrm{a}_{c}^{*}$, we shall denote by $\pi_{\xi, \nu}$ the principal series representation of $G$ induced by the representation $\operatorname{man} \mapsto \xi(m)^{-1} a^{-\rho-i p}$ of $M A N$ (see Wallach [18, §§8.3-8.4]).

2. Radon transform and differential operators. We use the notation of $\$ 1$. For $f \in \mathscr{D}(G)$, define

$$
\hat{f}(g, k)=\int_{N} f(g n k) d n, \quad g \in G, k \in K .
$$

Clearly $\hat{f} \in C^{\infty}(G \times K)$ and $\hat{f}(g m n, k)=\hat{f}(g, m k)$ for all $m \in M, n \in N$. This definition generalizes Helgason's Radon transform on the symmetric space $G / K$. It is also related to the principal series representations $\pi_{\xi, \nu}$, with $\xi \in \hat{M}, \nu \in a_{c}^{*}$, by the formula (see Wallach [18, \$8.8])

$$
\left(\pi_{\xi, \nu}(f) \varphi\right)\left(k^{\prime}\right)=\int_{M A} \xi(m) a^{\rho+i \nu} d m d a \int_{K} \hat{f}\left(k^{\prime} m a, k^{-1}\right) \varphi(k) d k,
$$

where $\varphi$ belongs to the space of $\pi_{\xi, \nu}$ and $k^{\prime} \in K$, and to its character by

$$
\operatorname{tr} \pi_{\xi, \nu}(f)=\int_{M A} \operatorname{tr} \xi(m) \cdot a^{\rho+i \nu} d m d a \int_{K} \hat{f}\left(k m a, k^{-1}\right) d k
$$

(tr denoting trace), which is the Fourier transform on $M A$ of the central function

$$
\tilde{f}(h)=\int_{K \times N} f\left(k h n k^{-1}\right) d k d n, \quad h \in M A
$$

(centrality follows from $\hat{f}\left(k h, m k^{-1}\right)=\hat{f}\left(k h m, k^{-1}\right)$ ). Also $f \mapsto \tilde{f}$ is continuous from $\mathscr{D}(G)$ into $\mathscr{D}(H)$.

2.1. Proposition (K. Johnson). Let $f \in \mathscr{D}(G)$ and $R>0$. The following are equivalent:

(i) $f(g)=0$ whenever $g \in G$ and $|g| \geqslant R$.

(ii) $\hat{f}(g, k)=0$ whenever $g \in G,\|g\| \geqslant R$ and $k \in K$.

PROOF. (i) implies (ii) easily, observing that $|g n k|=|g n|,\|g\|=\|g n\|$, and that for any $x \in G,|x|>\|x\|$ : by Iwasawa decomposition $x=k a n$, this inequality reduces to $|a n|>|a|$, which is true by Helgason [9, Lemma 5.4, p. 306].

(ii) implies (i). Let $\hat{K}$ denote the set of (equivalence classes of) irreducible unitary representations of $K, H_{\sigma}$ the (finite dimensional) space of $\sigma \in \hat{K}$. By Peter-Weyl theory on the compact group $K \times K, f$ can be decomposed as

$$
f(g)=\sum \operatorname{dim} \sigma \cdot \operatorname{dim} \tau \cdot \operatorname{tr} f_{\sigma \tau}(g), \quad \sigma, \tau \in \hat{K},
$$


with convergence in $\mathscr{D}(G)$; here

$$
f_{\sigma \tau}(g)=\int_{K \times K} f\left(k g k^{\prime}\right) \sigma(k)^{-1} \otimes \tau\left(k^{\prime}\right)^{-1} d k d k^{\prime}
$$

is $C^{\infty}$ on $G$, has compact support and values in the space $H_{o r}$ of all endomorphisms of $H_{\sigma} \otimes H_{\tau}$. Then (5) and (6) show that (i) holds for $f$ if and only if it holds for $f_{o \tau}$ for all $\sigma, \tau \in \hat{K}$; the same is true for (ii) in view of the formulas

$$
\left(f_{o \tau}\right)^{\wedge}(g, l)=\int_{K \times K} \hat{f}\left(k g, k^{\prime}\right) \sigma(k)^{-1} \otimes \tau\left(k^{\prime}\right)^{-1} \tau(l) d k d k^{\prime}
$$

and

$$
\hat{f}(g, l)=\sum \operatorname{dim} \sigma \cdot \operatorname{dim} \tau \cdot \operatorname{tr}\left(f_{\sigma \tau}\right)^{\wedge}(g, l) .
$$

Therefore it is enough to prove the proposition for each $f_{\sigma r}$. Now (ii) and the Paley-Wiener theorem on $M \times A$, i.e. the classical Paley-Wiener theorem on $A$, imply that for every integer $N>0$ there exists $C_{N}>0$ such that, for all $\xi \in \hat{M}, \nu \in a_{c}^{*}$,

$$
\left\|\int_{M A}\left(f_{o \tau}\right)^{\wedge}(m a, 1) \otimes \xi(m) a^{\rho-i \nu} d m d a\right\|<C_{N}(1+|\nu|)^{-N} e^{R|\operatorname{Im} \nu|},
$$

where \|\| is a norm on the tensor product of $H_{\sigma \tau}$ and the space of endomorphisms associated to $\xi$. By Johnson's Paley-Wiener theorem [13, Corollary 1, p. 25] this implies that $f_{\sigma \tau}(g)=0$ for $|g|>R$, which proves the proposition.

Proposition 2.1 generalizes to arbitrary functions in $\mathscr{D}(G)$ a result of Helgason for the $K$-bi-invariant case [9, Theorem 5.5, p. 306].

2.2. Remark. When $G$ is a complex semisimple Lie group, Proposition 2.1 can also be deduced from Želobenko's Paley-Wiener theorem. Reasoning on $f_{\text {or }}$, as above, one gets by (2),

$$
\left(\pi_{\xi, \nu}\left(f_{\sigma \tau}\right) \varphi\right)\left(k^{\prime}\right)=\sigma\left(k^{\prime}\right) \int_{M A}\left(f_{\sigma \tau}\right)^{\wedge}(m a, 1) \xi(m) a^{\rho+i \nu} d m d a \int_{K} \varphi(k) \tau\left(k^{-1}\right) d k
$$

(omitting the tensor product symbols) whence, using (7),

$$
\left\|\pi_{\xi, \nu}\left(f_{\sigma \tau}\right) \varphi\right\| \leqslant C_{N}(1+|\nu|)^{-N} e^{R|\operatorname{Im} \nu|}\|\varphi\|
$$

( $L^{2}(K)$-norms). This estimate of the operator norm of $\pi_{\xi, \nu}\left(f_{\sigma \tau}\right)$ implies that $f_{o \tau}(g)=0$ for $|g|>R$ by Želobenko [20, p. 1200].

In order to examine the action of bi-invariant differential operators on the Radon transform, we need an algebraic lemma.

If a Lie algebra $g$ is a vectorial direct sum $g=g^{\prime}+g^{\prime \prime}$ of two subalgebras then, as is well known, $U(\mathfrak{g})=U\left(\mathfrak{g}^{\prime}\right)+U(\mathfrak{g}) \mathfrak{g}^{\prime \prime}$ (direct sum of vector spaces). Applying this twice to the decomposition $\mathfrak{g}=\mathfrak{h}+\mathfrak{n}+\overline{\mathfrak{n}}$, with $\mathfrak{h}=\mathfrak{m}+\mathfrak{a}$, we get 


$$
U(\mathfrak{g})=U(\mathfrak{h})+U(\mathfrak{b}) \overline{\mathfrak{n}}+U(\mathfrak{g}) \mathfrak{n}
$$

with $\mathfrak{b}=\mathfrak{h}+\overline{\mathfrak{n}}$. Let $P=Q+R+S$ be the corresponding (unique) decomposition of $P \in U(\mathrm{~g})$, and consider the projections $\sigma, \tau$,

$$
\sigma(P)=Q+R, \quad \tau(P)=Q \text {. }
$$

Let $U(\mathfrak{g})_{\mathfrak{h}}$ be the centralizer of $\mathfrak{h}$ in $U(\mathfrak{g})$.

2.3. LEMMA. $\sigma$ and $\tau$ coincide on $U(\mathfrak{g})_{\mathfrak{h}}$ and define an algebra homomorphism of $U(\mathfrak{g})_{\mathfrak{h}}$ into $Z(\mathfrak{h})$.

(Cf. Dixmier [4, 7.4] for the case when $\mathfrak{h}$ is a Cartan subalgebra of $\mathfrak{g}$.)

For $X \in \mathfrak{h}$, ad $X$ maps $\mathfrak{h}$, resp. $\overline{\mathfrak{n}}, \mathfrak{b}, \mathfrak{n}$, into itself; therefore $\sigma \circ$ ad $X=$ ad $X \circ \sigma$ and $\tau \circ$ ad $X=\operatorname{ad} X \circ \tau$, whence $\tau\left(U(\mathfrak{g})_{\mathfrak{h}}\right) \subset Z(\mathfrak{h})$.

In order to prove that $\sigma-\tau$ is 0 on $U(g)_{\mathfrak{h}}$, it is therefore enough to show that the centralizer of $\mathfrak{h}$ in $U(\mathfrak{b}) \overline{\mathfrak{n}}$ is 0 . Let $\alpha$ be a positive (restricted) root of $\mathfrak{g}$ with multiplicity $m$, and select a basis $X_{1}, \ldots, X_{m}$ of $g_{-\alpha}$. Every multi-index $i=\left(i_{1}, \ldots, i_{m}\right) \in \mathbf{N}^{m}$ defines a standard monomial on $g_{-\alpha}, R^{i}=$ $X_{1}^{i_{1}} \ldots X_{m}^{i_{m}}$. Doing this for each positive root $\alpha_{1}, \ldots, \alpha_{r}$, and for $\alpha_{0}=0$, we see that any $R \in U(\mathfrak{b})$ can be uniquely written as

$$
R=\sum a_{j_{0}} \cdots j_{r} R_{0}^{j_{0}} \cdots R_{r}^{j_{r}}
$$

with complex $a$ 's, where $R_{0}^{j_{0}}, \ldots, R_{r}^{j_{r}}$ are standard monomials on $g_{0}=$ $\mathfrak{h}, \ldots, \mathfrak{g}_{-\alpha_{r}}$, defined by multi-indices $j_{0}, \ldots, j_{r}$; this holds in particular for $R \in U(\mathfrak{b}) \overline{\mathfrak{n}}$, with $j_{1}, \ldots, j_{r}$ never simultaneously vanishing. Now for $H \in \mathfrak{a}$, $\left[H, R^{i}\right]=-\alpha(H)|i| R^{i},|i|$ denoting the length of $i$, whence

$$
[H, R]=-\sum a_{j_{0}} \cdots j_{r}\left(\left|j_{1}\right| \alpha_{1}(H)+\cdots+\left|j_{r}\right| \alpha_{r}(H)\right) R_{0}^{j_{0}} \cdots R_{r}^{j_{r}} .
$$

If $R \in U(\mathfrak{b}) \bar{n}$ commutes to $\mathfrak{a}$, then by uniqueness

$$
a_{j_{0} \cdots j_{r}}\left(\left|j_{1}\right| \alpha_{1}(H)+\cdots+\left|j_{r}\right| \alpha_{r}(H)\right)=0
$$

for all $H \in \mathfrak{a}$; choosing $H$ so that $\alpha_{p}(H)>0,1 \leqslant p \leqslant r$, shows that $a_{j_{0}} \cdots j_{r}$ $=0$; therefore $R=0$ as claimed. Finally let $P, P^{\prime} \in U(g)_{b}$; then

$$
P P^{\prime}-\sigma(P) \sigma\left(P^{\prime}\right) \in \sigma(P) U(\mathfrak{g}) \mathfrak{n}+U(\mathfrak{g}) \mathfrak{n} \sigma\left(P^{\prime}\right)+U(\mathfrak{g}) \mathfrak{n} U(\mathfrak{g}) \mathfrak{n} .
$$

The first and third terms are contained in $U(\mathrm{~g}) \mathrm{n}$; the second too, for $\sigma\left(P^{\prime}\right)=\tau\left(P^{\prime}\right) \in U(\mathfrak{h})$ and $[U(\mathfrak{h}), \mathfrak{n}] \subset U(\mathfrak{h}) \mathfrak{n}$. This implies that $\sigma\left(P P^{\prime}\right)=$ $\sigma(P) \sigma\left(P^{\prime}\right)$, and proves the lemma.

2.4. REMARK. $\sigma$ and $\tau$ are not one-to-one on $U(\mathrm{~g})_{\mathfrak{h}}$ : the kernel $U(\mathrm{~g})_{\mathfrak{h}} \cap$ $U(\mathrm{~g}) \mathfrak{n}$ contains, for instance, $C-\sigma(C) \neq 0$, where $C$ is the Casimir element of $U(\mathrm{~g})$ (cf. $\S 5$, following). But they are when restricted to $Z(\mathrm{~g})$, since $Z(\mathrm{~g}) \cap U(\mathrm{~g}) \mathfrak{n}=0$ as follows from Lemma 2.3.3.5 in Warner [19, p. 167] applied to the complexification of $g$.

2.5. REMARK. Introducing the decompositions $\mathfrak{h}=\mathfrak{m}+\mathfrak{a}, U(\mathfrak{h})=U(\mathfrak{a})+$ $U(\mathfrak{h}) \mathrm{m}$, the corresponding projection $U(\mathfrak{h}) \rightarrow U(\mathfrak{a})$, and composing with $\tau$, we 
get a map $\chi: U(\mathfrak{g})_{\mathfrak{h}} \rightarrow U(\mathfrak{a})=Z(\mathfrak{a})$. For $P \in U(\mathfrak{g})_{\mathfrak{h}}$, one has

$$
P-\chi(P) \in U(\mathfrak{g}) \mathfrak{n}+U(\mathfrak{h}) \mathfrak{m} \subset \mathfrak{f} U(\mathfrak{g})+U(\mathfrak{g}) \mathfrak{n},
$$

since $[\mathfrak{m}, \mathfrak{h}] \subset \mathfrak{m} \subset \mathfrak{f}$. This shows that $\chi$ is closely related to Harish-Chandra's map $\gamma$ (cf. Helgason [7, Chapter X, §6.3]): extending the map $H \mapsto H_{\rho}=H$ $-\rho(H)$ to an automorphism of the symmetric algebra $S(\mathfrak{a})$, one has

$$
\gamma(P)=\chi(P)_{p}
$$

From now on, we shall deal only with the restriction of $\sigma$ and $\tau$ to the center, still denoted by

$$
\tau: Z(\mathfrak{g})=Z(G) \rightarrow Z(\mathfrak{h})=Z(M A) .
$$

2.6. Proposition. Let $P \in Z(G), f \in \mathscr{D}(G)$. Then

$$
(P f)^{\wedge}(g, k)=\tau(P)_{g} \hat{f}(g, k)=\tau(P)_{h} \hat{f}(g h, k)_{h=1}
$$

where $g \in G, k \in K, h \in M A$.

Proof. In view of right $G$-invariance of $P$ and right $N$-invariance of $\hat{f}$, it is clear that

$$
(P f)^{\wedge}(g, k)=P_{g} \hat{f}(g, k)=\sigma(P)_{g} \hat{f}(g, k),
$$

and the proposition follows from Lemma 2.3 .

With the notation of (4), we therefore have

$$
(P f)^{\sim}(h)=\tau(P)_{h} \tilde{f}(h), \quad h \in M A .
$$

Regarding $\tilde{f}$, we also prove a lemma which will be needed later.

2.7. Lemma. Let $h \in M A$, and $D(h)$ be the determinant of ( $\left.\operatorname{Ad~} h^{-1}-1\right)$ restricted to $\mathfrak{n}$. Then

$$
\tilde{f}(h)=|D(h)| \int_{G / A} f\left(g h g^{-1}\right) d \bar{g}
$$

when $f \in \mathscr{D}(G)$ and $D(h) \neq 0$ (here d $\bar{g}$ is a suitably normalized G-invariant measure on $G / A$, and the integral is absolutely convergent).

Proof. When $D(h) \neq 0$ it is known (Wallach [18, p. 174]) that the map $\varphi$ : $n \mapsto h^{-1} n h n^{-1}$ is a diffeomorphism of $N$ onto itself with jacobian $D(h)$, whence

$$
\int_{N} F(n) d n=|D(h)| \int_{N} F\left(h^{-1} n h n^{-1}\right) d n, \quad F \in \mathscr{D}(N) .
$$

Applying this to $F(n)=f\left(k h n k^{-1}\right)$ we get

$$
\tilde{f}(h)=|D(h)| \int_{K \times N} f\left(k n \cdot h \cdot(k n)^{-1}\right) d k d n .
$$

On the other hand the $A$-invariant function $g \mapsto f\left(g h g^{-1}\right)$ defines a function 
$u \in \mathscr{D}(G / A)$ (compactness of support follows from $\varphi$ being proper, writing $g=k n a)$, and the formula

$$
\int_{G / A} u(g A) d \bar{g}=\int_{K \times N} u(k n A) d k d n
$$

implies the lemma.

2.8. Proposition. Let $P \in Z(G)$. Then $\tau(P)$-convexity of $H=M A$ implies $P$-convexity of $G$.

Recall that $G$ is said to be $P$-convex if for any compact subset $C$ of $G$ there exists a compact $C^{\prime}$ such that, for any $T \in \mathcal{E}^{\prime}(G)$, supp $P^{*} T \subset C$ implies supp $T \subset C^{\prime}$, or equivalently by regularization, for any $f \in \mathscr{D}(G)$, supp $P^{*} f$ $\subset C$ implies supp $f \subset C^{\prime}$; for bi-invariant operators, $P$-convexity of $G$ is equivalent to $P^{*}$-convexity of $G$, in view of Lemma 1.1. Analogously on $H$.

Proof. Let $f \in \mathscr{D}(G)$ and assume $P f(x)=0$ for $|x|>R$. Then, using 2.1 and 2.6,

$$
(P f)^{\wedge}\left(k h, k^{\prime}\right)=\tau(P)_{h} \hat{f}\left(k h, k^{\prime}\right)
$$

vanishes for $k, k^{\prime} \in K, h \in H,|h| \geqslant R$. By $\tau(P)$-convexity of $H$ there exists $R^{\prime}>0$ such that all functions $h \mapsto \hat{f}\left(k h, k^{\prime}\right)$, which belong to $\mathscr{D}(H)$, vanish for $|h|>R^{\prime}$. Owing to right $N$-invariance it follows that $\hat{f}\left(x, k^{\prime}\right)=0$ for $x \in G,\|x\|>R^{\prime}, k^{\prime} \in K$, therefore $f(x)=0$ for $x \in G,|x|>R^{\prime}$ by 2.1, which proves the proposition.

3. Invariant differential operators on $M \times A$. In this section, $M$ denotes any compact connected Lie group, $A$ any Lie group which is isomorphic to the additive group of a vector space, and $H=M \times A$ their direct product. $\mathfrak{h}$, $\mathfrak{m}, \mathfrak{a}$ are the Lie algebras.

Let $H_{\xi}$ be the complex Hilbert space on which acts $\xi \in \hat{M}$, and $L\left(H_{\xi}\right)$ its space of endomorphisms. For $Q \in D(H), \xi \in \hat{M}$, we define $Q(\xi) \in D(A)$ $\otimes L\left(H_{\xi}\right)$ by

$$
Q(\xi)_{a} f(a)=\xi(m)^{-1} Q_{m a}(\xi(m) f(a)), \quad f \in C^{\infty}(A),
$$

and, for $\nu \in a_{c}^{*}, Q(\xi, \nu) \in L\left(H_{\xi}\right)$ by

$$
Q(\xi, \nu)=a^{-\nu} Q(\xi)\left(a^{\nu}\right)=\xi(m)^{-1} a^{-\nu} Q\left(\xi(m) a^{\nu}\right) .
$$

If $Q$ is written $\Sigma a_{\alpha \beta} X^{\alpha} Y^{\beta}$ with respect to a basis $X_{1}, \ldots, X_{p}, Y_{1}, \ldots, Y_{n}$ of $\mathfrak{m}+\mathfrak{a}$, then

$$
Q(\xi, \nu)=\sum a_{\alpha \beta}(\xi(X))^{\alpha}(\nu(Y))^{\beta},
$$

where $\xi(X)=X \xi(1)$ (we keep the same symbol $\xi$ for representations of $M$ and $\mathfrak{m})$.

3.1. Proposition. Let $Q \in D(H)$. The following are equivalent: 
(i) $H$ is $Q$-convex;

(ii) for any $\xi \in \hat{M}$, det $Q^{*}(\xi, \nu)$, as a polynomial in $\nu$, is not identically zero.

(Cf. Johnson's injectivity criterion [13, p. 28].) When $Q \in Z(H)$, (ii) can be replaced by: for any $\xi \in \hat{M}, Q(\xi, \nu)$ is not identically zero. In fact, $Q(\xi, \nu)$ is then a scalar and $Q$-convexity is equivalent to $Q^{*}$-convexity by Lemma 1.1 .

Proof. Let $f \in \mathscr{D}(H)$ and $C$ be a compact subset of $A$. Considering the partial Fourier transform

$$
\hat{f}(\xi, a)=\int_{M} \xi(m)^{-1} f(m a) d m, \quad a \in A, \xi \in \hat{M},
$$

it is immediate that supp $f \subset M \times C$ if and only if supp $\hat{f}(\xi, \cdot) \subset C$ for all $\xi \in \hat{M}$, and that

$$
\left(Q^{*} f\right)^{\wedge}(\xi, a)=Q^{*}(\xi) \hat{f}(\xi, a) .
$$

(i) implies (ii). If det $Q^{*}(\eta, \nu) \equiv 0$ for some $\eta \in \hat{M}$, there exists a nonzero polynomial mapping $\nu \mapsto R(\nu) \in L\left(H_{\eta}\right)$ such that $Q^{*}(\eta, \nu) R(\nu) \equiv 0$. Let $R \in D(A)$ be the corresponding differential operator, according to (14); one has $Q^{*}(\eta) R=0$. Taking $\varphi \in \mathscr{D}(A), \varphi \neq 0$, we set

$$
f(m a)=\operatorname{dim} \eta \cdot \operatorname{tr}(\eta(m) R \varphi(a)) .
$$

Then $\hat{f}(\xi, a)=0$ for $\xi \neq \eta$ and $\hat{f}(\eta, a)=R \varphi(a)$, so that $f \in \mathscr{D}(H)$ is a nontrivial solution of $Q^{*} f=0$; consequently, $H$ is not $Q$-convex.

(ii) implies (i). Let $C$ be a compact convex subset of $A$, and $f \in \mathscr{D}(H)$ such that supp $Q^{*} f \subset M \times C$; then $\operatorname{supp} Q^{*}(\xi) \hat{f}(\xi, \cdot) \subset C$ for all $\xi$. Applying the transpose of the matrix of cofactors of $Q^{*}(\xi)$ it follows that supp (det $\left.Q^{*}(\xi) \hat{f}(\xi, \cdot)\right) \subset C$; by the classical theory of constant coefficient differential operators this implies $\operatorname{supp} \hat{f}(\xi, \cdot) \subset C$, in view of (ii), whence the conclusion.

In order to state the next result, we need some more notation. Since $M$ is compact, we have $\mathfrak{m}=\mathfrak{m}_{0}+\mathfrak{m}^{\prime}$, where $\mathfrak{m}_{0}$ is the center of $\mathfrak{m}$ and $\mathfrak{m}^{\prime}=[\mathfrak{m}, \mathfrak{m}]$ is semisimple. Let us take a positive definite inner product on $\mathfrak{m}$ extending the opposite of the Killing form of $\mathfrak{m}^{\prime}$ and such that $\mathfrak{m}_{0}$ is orthogonal to $\mathfrak{m}^{\prime}$; let $X_{1}, \ldots, X_{p}$ be an orthonormal basis of $\mathrm{m}$. Then $\Delta=\Sigma X_{k}^{2}$ belongs to $Z(M)$ and we set

$$
d(\xi)=1-\Delta(\xi)=1-\sum \xi\left(X_{k}\right)^{2}, \quad \xi \in \hat{M} ;
$$

$d(\xi)$ is a strictly positive scalar, which plays the rôle of a "norm" for $\xi$ (cf. also Wallach [18, §5.6]); when $M$ is abelian $\hat{M}$ can be identified with $\mathbf{Z}^{p}$, and $d(\xi)=1+|\xi|^{2}$.

3.2. Proposition. Let $Q \in Z(H)$. The following are equivalent:

(i) $Q$ has a fundamental solution; 
(ii) there exist $C>0$ and an integer $k \geqslant 0$ such that $\|Q(\xi, \cdot)\| \geqslant C d(\xi)^{-k}, \quad$ for all $\xi \in \hat{M}$.

(Here \|| $\|$ is any norm on the space of polynomials of degree $<m$, say, in $\nu \in a_{c}^{*}$.)

Proof. We refer to $[2, \S 7]$ for a complete proof, and for a more general statement valid when $Q \in D(H)$. For the reader's convenience we shall only sketch here a simpler proof of (ii) implies (i). Let $m$ be a positive integer. Then, by the classical theory of differential operators with constant coefficients (cf. Hörmander [12, \$3.1]), there exists a continuous norm III II| on $\mathscr{D}(A)$ such that, for all $u \in \mathscr{D}(A)$ and all $P \in D(A)$ with degree $\leqslant m$,

$$
\|P(\cdot)\| \cdot|u(1)| \leqslant\|P u\| \mid
$$

(this inequality is an easy consequence of (3.1.11) in [12]). Applying this to $P=Q(\xi)^{*}$ (transpose in $D(A)$ ), which corresponds to the polynomial $P(\nu)=$ $Q(\xi,-\nu)$, we get by (ii),

$$
|u(1)| \leqslant C d(\xi)^{k}||\left|Q(\xi)^{*} u\right|||
$$

with $C$ independent of $\xi$ and $u$. It follows from the Hahn-Banach theorem that $Q(\xi)$ admits a fundamental solution $E_{\xi} \in D^{\prime}(A)$ such that

$$
\left|\left\langle E_{\xi}, u\right\rangle\right| \leqslant C d(\xi)^{k}|\|u \mid\| \text {. }
$$

Now these inequalities imply that the series

$$
\sum \operatorname{dim} \xi \cdot \operatorname{tr} \xi(m) \cdot E_{\xi}(a) \quad(\xi \in \hat{M})
$$

converges in $\mathscr{D}^{\prime}(H)$ and define a distribution $E(m, a)$ such that $Q E=\delta_{H}$, which proves our claim.

3.3. Remark. Propositions 3.1 and 3.2 show that, for $Q \in Z(H)$ (in fact for all $Q \in D(H)$ ), the existence of a fundamental solution implies $Q C^{\infty}(H)$ $=C^{\infty}(H)$.

4. Global solvability on $G$. We now return to the notation of $\S 1$. We begin with a necessary condition for global solvability, involving Harish-Chandra's map $\gamma$ defined in 2.5.

Let $\mathscr{D}_{K}(G)$, resp. $C_{K}^{\infty}(G)$, be the space of left and right $K$-invariant functions in $\mathscr{D}(G)$, resp. $C^{\infty}(G), \mathscr{D}_{K}^{\prime}(G)$ the dual of $\mathscr{D}_{K}(G)$, identified with the space of left and right $K$-invariant distributions in $\mathscr{D}^{\prime}(G)$, and similarly $\mathscr{D}_{W}(A)$, resp. $\mathscr{D}_{W}^{\prime}(A)$, the space of $W$-invariant elements of $\mathscr{D}(A)$, resp. D) $(A)$. Let

$$
F f(a)=a^{\rho} \int_{N} f(a n) d n=a^{\rho} \tilde{f}(a),
$$

$f \in \mathscr{D}_{K}(G), a \in A$; then $f \mapsto F f$ is an isomorphism of $\mathscr{D}_{K}(G)$ onto $\mathscr{D}_{W}(A)$ 
for the Schwartz topologies (Helgason [9], Gangolli [5]); for $P \in Z(G)$, $F(P f)=\gamma(P) F f$, as a consequence of (9) and (11), and $\gamma$ defines an algebra homomorphism from $Z(G)$ into $Z(A)$. Transposing $F^{-1}$ we get an isomorphism $F^{*}$ of $\mathscr{D}_{K}^{\prime}(G)$ onto $\mathscr{D}_{W}^{\prime}(A)$, and

$$
F^{*}(P T)=\left(\gamma\left(P^{*}\right)\right)^{*} F^{*} T, \quad P \in Z(G), T \in \mathscr{D}_{K}^{\prime}(G) .
$$

We also mention the formula

$$
\gamma(P)(\nu)=\tau(P)\left(\xi_{0}, \nu-\rho\right), \quad \nu \in \mathfrak{a}_{c}^{*},
$$

with notation of (13), $\xi_{0} \in \hat{M}$ denoting the trivial representation; indeed $\gamma(P)(\nu)=\chi(P)(\nu-\rho)$ by $(9)$, and $\tau(P)-\chi(P)$ annihilates the function $\xi_{0}(m) a^{\nu-\rho}=a^{\nu-\rho}$, since it belongs to $U(\mathfrak{h}) m$ by definition of $\chi$.

4.1. Proposition. Let $P \in Z(G)$. If $\gamma(P)=0$, then $P$ is not globally solvable, has no parametrix, and no index in $C^{\infty}(G)$.

Globally solvable means that $P \mathscr{D}^{\prime}(G) \supset \mathscr{D}(G)$, and recall that a parametrix is a distribution $E \in \mathbb{D}^{\prime}(G)$ such that $P E-\delta_{G} \in C^{\infty}(G)$. The kernel of $\gamma$ restricted to $Z(G)$ is $Z(G) \cap D(G) \mathfrak{f}$ (Helgason [7, p. 431]); it consists of those operators in $Z(G)$ which induce 0 on the symmetric space $G / K$.

Proof. First assume $P$ is globally solvable; it would even be enough to assume $P \Phi^{\prime}(G) \supset \mathscr{D}_{K}(G)$. Making solutions $K$-invariant we get $P \mathscr{D}_{K}^{\prime}(G) \supset$ $\mathscr{D}_{K}(G)$, then $P^{*} \mathscr{D}_{K}^{\prime}(G) \supset \mathscr{D}_{K}(G)$ by the diffeomorphism $x \mapsto x^{-1}$ together with Lemma 1.1. Applying $F^{*}$ yields (by (16)) $\gamma(P)^{*} \mathscr{D}_{W}^{\prime}(A) \supset F^{*} \mathscr{D}_{K}(G)$; therefore $\gamma(P) \neq 0$.

When $P$ has a parametrix $E$, then $P^{*}$ too, and we get $P^{*} E_{K}-\delta_{K} \in$ $C_{K}^{\infty}(G)$ making everything $K$-bi-invariant. Applying $F^{*}$ yields $\gamma(P)^{*} F^{*} E_{K}-$ $F^{*} \delta_{K} \in F^{*} C_{K}^{\infty}(G)$; now $\gamma(P)=0$ would imply $\delta_{K} \in C_{K}^{\infty}(G)$, which is absurd.

Finally when $\gamma(P)=0$ the kernel of $P$ in $C^{\infty}(G)$ contains the infinite dimensional subspace $C_{K}^{\infty}(G)$; on the other hand, $C_{K}^{\infty}(G) \cap P C^{\infty}(G)=$ $P C_{K}^{\infty}(G)=\{0\}$; therefore $P C^{\infty}(G)$ is infinite codimensional in $C^{\infty}(G)$. It follows that $P$ has no index in $C^{\infty}(G)$, whence the proposition.

From now on, we make the further assumption that $G$ has one conjugacy class of Cartan subalgebras.

We refer to Helgason [10, p. 45] and Wallach [18, \$7.9] for some properties of these groups, and we shall only recall here the following inversion formula: there exists $R$, a differential operator with $C^{\infty}$ coefficients in a neighborhood of 1 in $M A$, such that for any $f \in \mathscr{D}(G)$,

$$
f(1)=R \tilde{f}(1),
$$

with $\tilde{f}(m a)=\int_{K \times N} f\left(k_{m a n k}{ }^{-1}\right) d k d n$ (see Wallach [18, §7.11], for an explicit formula for $R$, and Guillemin [6] for a proof using stationary phase methods 
when $G$ is complex). $R$ will be used at 1 only; we can therefore freeze the coefficients and assume $R \in D(H)$. Furthermore, since $\tilde{f}$ is a central function on $H$, we can replace $R$ by $\int_{M} \operatorname{Ad}(m) R \cdot d m$, which is a bi-invariant operator on $H=M A$. Thus we shall take $R \in Z(H)$ in (18).

By translation, (18) gives an inversion formula for the Radon transform (1): let $g=K(g) A(g) N(g)$ be the Iwasawa decomposition of $g \in G$; then for $x \in G, k \in K, m \in M, a \in A$,

$$
x k m a=K(x k m) A(x k m) N(x k m) a=K(x k) m A(x k) a n
$$

with $n \in N$ depending on $x, k, m, a$, so that for $f_{x}(g)=f(x g)$ we have

$$
\left(f_{x}\right)^{\sim}(h)=\int_{K} \hat{f}\left(K(x k) A(x k) h, k^{-1}\right) d k, \quad h \in M A,
$$

whence

$$
f(x)=\int_{K}\left(R_{h} \hat{f}\left(K(x k) A(x k) h, k^{-1}\right)\right)_{h=1} d k .
$$

This formula extends Helgason's inversion formula for the Radon transform on $G / K[10$ p. 43]. Let us note that (19) shows our Radon transform $f \mapsto \hat{f}$ is one-to-one from $\mathscr{D}(G)$ into $C^{\infty}(G \times K)$, which implies by (10) that $\tau: Z(G)$ $\rightarrow Z(H)$ is one-to-one (cf. Remark 2.4). However Proposition 2.1 is not an immediate consequence of (19), since, for $x \in A, \log A(x k)$ runs through the whole convex hull of the Weyl group orbit of $\log x$, as $k$ runs through $K$ (Kostant [14, p. 433]).

We now come to the main result of this paper.

4.2. TheOREM. Let $G$ be a connected semisimple Lie group with one conjugacy class of Cartan subalgebras, and $P \in Z(G)$. Assume $\tau(P)$ has a fundamental solution, as a differential operator on $H=M A$. Then $P$ has a fundamental solution on $G$, which is invariant under inner automorphisms, and $P C^{\infty}(G)=$ $C^{\infty}(G)$.

$\tau(P) \in Z(H)$ is defined in $\$ 2$, and an explicit criterion on the Fourier coefficients of $\tau(P)$ is given by 3.2(ii).

Proof. By Lemma 1.1 we can replace $P$ and $\tau(P)$ by the transpose operators. Let $T \in \Phi^{\prime}(H)$ be a fundamental solution of $\tau(P)^{*}$, and set

$$
\langle E, f\rangle=\langle T, R \tilde{f}\rangle, \quad f \in \mathscr{D}(G),
$$

with $R \in Z(H)$ as in (18). This defines $E \in \mathscr{D}^{\prime}(G)$ such that $P^{*} E=\delta_{G}$. Indeed

$$
\begin{aligned}
\left\langle P^{*} E, f\right\rangle & =\langle E, P f\rangle=\langle T, R \tau(P) \tilde{f}\rangle \quad \text { by }(11) \\
& =\langle T, \tau(P) R \tilde{f}\rangle=\left\langle\tau(P)^{*} T, R \tilde{f}\right\rangle \\
& =R \tilde{f}(1)=f(1) \quad \text { by }(18) .
\end{aligned}
$$


In order to prove $E$ is central it suffices, by (20), to show that $\left(f^{g}\right)^{\sim}(h)=\tilde{f}(h)$, letting $f^{g}(x)=f\left(g x g^{-1}\right)(g \in G, x \in G, h \in H)$. This follows from Lemma 2.7 when $D(h) \neq 0$; the same holds for all $h \in H$ by density; it can be obtained by representation theory also, through (3). Now $P$, having a fundamental solution, is "semiglobally solvable" on $G$, i.e. for every $f \in C^{\infty}(G)$ and every relatively compact open subset $\Omega$ of $G$, there exists $u \in C^{\infty}(G)$ such that $P u=f$ on $\Omega$. Furthermore, $H$ is $\tau(P)$-convex by Propositions 3.1 and 3.2, and it follows that $G$ is $P$-convex by Proposition 2.8. From Theorem 1.9 in Treves [17, p. 106], we conclude that $P C^{\infty}(G)=C^{\infty}(G)$; this proves the theorem.

5. Examples and counterexamples. We keep to the notation of $\S 1$, assuming that $G$ has one conjugacy class of Cartan subalgebras. For $P \in Z(G)$ we shall denote by (A), (B), (C) the conditions of Propositions 4.1, 3.1, 3.2:

(A) $\gamma(P) \neq 0$;

(B) for all $\xi \in \hat{M},\|\tau(P)(\xi, \cdot)\| \neq 0$;

(C) there exists $C>0$ and an integer $k \geqslant 0$ such that, for all $\xi \in \hat{M}$, $\|\tau(P)(\xi, \cdot)\| \geqslant C d(\xi)^{-k}$.

(a) The Casimir operator. Let $B$ be an invariant symmetric nondegenerate bilinear form on a Lie algebra $\mathfrak{l}, L_{1}, \ldots, L_{n}$ a basis of $\mathfrak{l},\left(a_{j k}\right)$ the inverse matrix of $\left(B\left(L_{j}, L_{k}\right)\right)$; then

$$
C=\sum a_{j k} L_{j} L_{k} \quad(1 \leqslant j, k \leqslant n)
$$

defines the corresponding Casimir element $C \in Z(\mathfrak{l})$, which is independent of the choice of basis $\left(L_{j}\right)$. Let us take $\mathfrak{l}=\mathfrak{g}=\mathfrak{m}+\mathfrak{a}+\mathfrak{n}+\overline{\mathfrak{n}}$ with the Killing form, $X_{1}, \ldots, X_{p}$ an orthonormal basis of $\mathfrak{m}$ with respect to $-B$, $Y_{1}, \ldots, Y_{q}$ an orthonormal basis of $a$ with respect to $B$, and $Z_{1}, \ldots, Z_{r}$ an orthonormal basis of $\mathfrak{n}$ with respect to the scalar product $\langle X, Y\rangle=$ $-B(X, \theta Y)$. With the $X, Y, Z$, and $\theta Z$ as a basis of $g$, an easy computation shows that

$$
C=-\sum_{1}^{p} X_{i}^{2}+\sum_{1}^{q} Y_{j}^{2}-\sum_{1}^{r}\left(Z_{k} \cdot \theta Z_{k}+\theta Z_{k} \cdot Z_{k}\right)
$$

therefore $C=C^{*}$. To compute $\tau(C)$ we write $C$ as

$$
C=-\sum X_{i}^{2}+\sum Y_{j}^{2}+H-2 \sum \theta Z_{k} \cdot Z_{k},
$$

with $H=\Sigma\left[\theta Z_{k}, Z_{k}\right]$. But the $U(\mathfrak{b}) \overline{\mathfrak{n}}$-component of $C$ is 0 by Lemma 2.3; therefore $H \in \mathfrak{m}+\mathfrak{a}+\mathfrak{n}$; since $\theta H=-H$ obviously, and the sum $\mathfrak{m}+a$ $+\mathfrak{n}+\theta \mathfrak{n}$ is direct, it follows that $H \in \mathfrak{a}$. That implies

$$
\tau(C)=-\sum X_{i}^{2}+\sum Y_{j}^{2}+H,
$$

and, with the notation of (13), (14), 


$$
\tau(C)(\xi, \nu)=-\sum \xi\left(X_{i}\right)^{2}+\sum \nu\left(Y_{j}\right)^{2}+\nu(H)
$$

$\left(\xi \in \hat{M}, \nu \in \mathrm{a}_{c}^{*}\right)$. The above computations are, of course, valid for any real semisimple Lie algebra.

Let $p$ be any nonzero polynomial in one variable with complex coefficients; it is now clear that in $\tau(p(C))(\xi, \nu)=p(\tau(C)(\xi, \nu))$, the terms of highest degree in $\nu$ come from $p\left(\sum \nu\left(Y_{j}\right)^{2}\right)$, and they do not depend on $\xi$. Thus (C) is satisfied with $k=0$. We conclude from Theorem 4.2:

5.1. Corollary. Let $G$ be a connected semisimple Lie group with one conjugacy class of Cartan subalgebras, $P$ any nonzero polynomial of the Casimir operator. Then $P$ has a central fundamental solution, and $P C^{\infty}(G)=C^{\infty}(G)$.

5.2. ReMARKs. For general semisimple Lie groups, Rauch and Wigner [15] have proved by entirely different methods that $C C^{\infty}(G)=C^{\infty}(G)$.

As regards fundamental solutions, Benabdallah [1] has shown that $C$ has one when $G=\operatorname{SL}(2, \mathbf{R})$ (cf. [16] also), but none is central because of difficulties coming from the discrete series. Let us mention that our method becomes similar to Benabdallah's when expressed in terms of characters of $G$ instead of the Radon transform: Assume $G$ complex for simplicity, and let us restrict to operators $P$ such that $\left|\tau\left(P^{*}\right)^{*}(\xi, \rho+i v)\right|>C>0$ for every $\xi \in$ $\hat{M}, \nu \in a^{*}$. The Plancherel formula (18) can be written as

$$
\delta_{G}=\sum_{\xi \in \hat{M}} \int_{\mathfrak{a}^{*}} \chi_{\xi, \nu} \cdot r(\xi, \nu) d \nu,
$$

a decomposition of the Dirac measure according to the characters $\chi_{\xi, \nu}=$ $\operatorname{tr} \pi_{\xi, \nu} \in \mathscr{D}^{\prime}(G)$ (here $r(\xi, \nu)$ is a polynomial corresponding to $R$ ). To solve $P E=\delta_{G}$ it is therefore natural to look for distributions $T_{\xi, \nu}$ such that $P T_{\xi, \nu}=\chi_{\xi, \nu}$. But

$$
P \chi_{\xi, \nu}=\tau\left(P^{*}\right)^{*}(\xi, \rho+i v) \chi_{\xi, \nu}
$$

by (3), (11), (13), and our assumptions allow us to take

$$
T_{\xi, \nu}=\tau\left(P^{*}\right)^{*}(\xi, \rho+i v)^{-1} \chi_{\xi, \nu}
$$

and

$$
E=\sum \int T_{\xi, \nu} r(\xi, \nu) d \nu
$$

that agrees with (20) and clearly shows $E$ is central.

(b) The "imaginary" Casimir operator. Assume $G$ complex. Then its Lie algebra $g$ (over R) admits a complex structure $J\left(J^{2}=-1, J \circ\right.$ ad $X=$ $\operatorname{ad} X \circ J=\operatorname{ad}(J X))$, giving rise to a new symmetric nondegenerate invariant form

$$
B_{J}(X, Y)=-B(X, J Y)
$$


therefore to the "imaginary" Casimir operator $C_{J} \cdot \frac{1}{2} B$ and $\frac{1}{2} B_{J}$ are real and imaginary part, respectively, of the Killing form of the complex Lie algebra $\mathrm{g}$.

Let $U_{1}, \ldots, U_{n}$ be a basis of $\mathfrak{f}$, orthonormal with respect to $-B$, and $V_{j}=-J U_{j}$; then the $U$ and $V$ are a basis of $\mathfrak{g}=\mathfrak{f}+J \mathfrak{f}$ (over $\mathbf{R}$ ) and the corresponding expressions of $C$ and $C_{J}$ are

$$
\begin{aligned}
& C=-\sum U_{j}^{2}+\sum V_{j}^{2} \\
& C_{J}=2 \sum U_{j} V_{j}=2 \sum V_{j} U_{j}
\end{aligned}
$$

5.3. Proposition. ( $G$ complex). Let $p$ be any polynomial in one variable, with complex coefficients, such that $p(0)=0$. Then $p\left(C_{J}\right)$ is not globally solvable, has no parametrix, and no index in $C^{\infty}(G)$.

This is an immediate consequence of Proposition 4.1, since $\gamma\left(p\left(C_{J}\right)\right)=$ $p\left(\gamma\left(C_{J}\right)\right)$ and $C_{J} \in Z(G) \cap D(G) \mathfrak{f}$, which is the kernel of $\gamma$.

Let us point out that the method of [15] does not apply to $C_{J}$ since it has null bicharacteristic strips staying over $K$ : the principal symbol at 1 is $\sum \eta\left(U_{j}\right) \eta\left(V_{j}\right)$ ( $\eta$ a linear form on $g$ ) and the bicharacteristic strip through $(1, \eta)$ projects on $G$ as

$$
x(t)=\exp \left(t \sum\left(\eta\left(V_{j}\right) U_{j}+\eta\left(U_{j}\right) V_{j}\right)\right)
$$

(cf. [16, p. 853]); therefore stays over $K$ if $\eta$ vanishes on $f$.

When $G$ is complex we can give for $\tau(C)$ a more specific formula than (23). As above we use the Cartan decomposition $\mathfrak{g}=\mathfrak{f}+J \mathfrak{E}$, so that $\theta J=-J \theta$. If $\mathfrak{a}$ is maximal abelian in $J \mathfrak{f}$, then $\mathfrak{m}=J \mathfrak{a}$, and $\mathfrak{h}=\mathfrak{m}+\mathfrak{a}$ is a Cartan subalgebra of $g$. For any $\mathbf{R}$-vector space $V$ with complex structure, we denote by $\tilde{V}$ the corresponding $\mathbf{C}$-vector space. The roots of the pair $(\tilde{\mathfrak{g}}, \tilde{\mathfrak{h}})$ are the complex extensions $\tilde{\alpha}$ of the roots $\alpha$ of $(\mathfrak{g}, \mathfrak{a})$ and, for the root spaces, $\tilde{\mathfrak{g}}_{\tilde{\alpha}}=\tilde{\mathfrak{g}}_{\alpha}$ (cf. Helgason [7, Chapter VI, §6]). The (real) multiplicities are 2, and $\rho=\Sigma \alpha$ $(\alpha>0)$. Let $\tilde{B}$ be the Killing form of $\tilde{\mathrm{g}}$, so that $B=2 \operatorname{Re} \tilde{B}, B_{J}=2 \operatorname{Im} \tilde{B}$. We choose a basis $Y_{1}, \ldots, Y_{p}$ of $a$ over $R$, orthonormal with respect to $B$, and, for each $\alpha>0$, a vector $Z_{\alpha} \in \tilde{g}_{\alpha}$ such that $\tilde{B}\left(Z_{\alpha}, \theta Z_{\alpha}\right)=-\frac{1}{2}$; we set $Z_{\alpha}^{\prime}=J Z_{\alpha}$ and $X_{j}=J Y_{j}, 1<j<n$. Then $\left(X_{j}\right)$ is an orthonormal basis of $m$ with respect to $-B$, and $\left(Z_{\alpha}, Z_{\alpha}^{\prime}\right), \alpha>0$, an orthonormal basis of $\mathfrak{n}$ with respect to $-B(X, \theta Y)$. Now, defining $H_{\alpha} \in \tilde{\mathfrak{h}}$ by $\tilde{B}\left(H, H_{\alpha}\right)=\frac{1}{2} \tilde{\alpha}(H)$ for all $H \in \tilde{\mathfrak{h}}$, it is easy to show $H_{\alpha} \in \mathfrak{a}$ and $\left[\theta Z_{\alpha}, Z_{\alpha}\right]=H_{\alpha}=\left[\theta Z_{\alpha}^{\prime}, Z_{\alpha}^{\prime}\right]$, whence, according to (22),

$$
C=-\sum X_{j}^{2}+\sum Y_{j}^{2}+2 H_{\rho}-2 \sum_{\alpha>0}\left(\theta Z_{\alpha} \cdot Z_{\alpha}+\theta Z_{\alpha}^{\prime} \cdot Z_{\alpha}^{\prime}\right) .
$$

Let $\Gamma$ be the unit lattice of $M$, i.e. the set of $X \in J \mathfrak{a}$ such that $\exp X=1$. Then, in order to avoid imaginary numbers, we shall identify $\hat{M}$ with the 
lattice $\Gamma^{*}$ of the $\xi \in a^{*}$ such that $\xi(J \Gamma) \subset 2 \pi \mathrm{Z}$ (such a $\xi$ defines the representation $\left.\pi(\exp X)=e^{i \xi(-J X)}, X \in \mathfrak{m}\right)$. We finally obtain

$$
\tau(C)(\xi, \nu)=\sum\left(\xi_{j}^{2}+\nu_{j}^{2}+2 \rho_{j} \nu_{j}\right)
$$

$(1<j<p)$ with $\xi \in \Gamma^{*}, \nu \in a_{c}^{*}$, and for $\lambda \in a_{c}^{*}, \lambda_{j}=\lambda\left(Y_{j}\right)$. Also

$$
\gamma(C)(\nu)=\sum\left(\nu_{j}^{2}-\rho_{j}^{2}\right)
$$

by (17), and

$$
C \chi_{\xi, \nu}=\sum\left(\xi_{j}^{2}-\nu_{j}^{2}-\rho_{j}^{2}\right) \chi_{\xi, \nu}
$$

with $\Sigma \rho_{j}^{2}=\rho\left(H_{\rho}\right)$. Similarly,

$$
\begin{gathered}
C_{J}=\sum X_{j} Y_{j}+2 J H_{\rho}+2 \sum_{\alpha>0}\left(\theta Z_{\alpha}^{\prime} \cdot Z_{\alpha}-\theta Z_{\alpha} \cdot Z_{\alpha}^{\prime}\right) \\
\tau\left(C_{J}\right)(\xi, \nu)=i \sum \xi_{j}\left(\nu_{j}+2 \rho_{j}\right) \\
\gamma\left(C_{J}\right)(\nu)=0, \quad C_{J} \chi_{\xi, \nu}=i \sum \xi_{j}\left(i \nu_{j}-\rho_{j}\right) \chi_{\xi, \nu}
\end{gathered}
$$

We now examine the relations between (A), (B), (C) at the beginning of this section. Obviously (C) implies (B) and (B) implies (A). The following generalization of 5.1 and 5.3 gives a partial converse.

5.4 Proposition. Assume $G$ complex, with split rank $>2$, and let $P$ belong to the subalgebra of $Z(G)$ generated by $C$ and $C_{J}$. Then $P$ has a fundamental solution, or $P C^{\infty}(G)=C^{\infty}(G)$, if and only if $\gamma(P) \neq 0$. (The split rank of $G$ is the dimension of a (over $\mathbf{R})$.)

Proof. Necessity follows from Proposition 4.1. For the sufficiency we shall prove that (A) and (C) are equivalent for $P$; the conclusion will be given by Theorem 4.2.

(A) implies (B) for $P$. Let $P=p\left(C, C_{J}\right), p$ a polynomial in two variables with complex coefficients. Since $\operatorname{dim} a \geqslant 2, \Sigma\left(\nu_{j}^{2}+2 \rho_{j} \nu_{j}+\xi_{j}^{2}\right)$ and $\Sigma\left(\xi_{j} \nu_{j}+\right.$ $2 \xi_{j} \rho_{j}$ ) are algebraically independent, as polynomials in $\nu \in a_{c}^{*}$, for any $\xi \in \Gamma^{*}$, $\xi \neq 0$. Thus we see by (24), (26) that

$$
\tau(P)(\xi, \nu)=p\left(\tau(C)(\xi, \nu), \tau\left(C_{J}\right)(\xi, \nu)\right)
$$

can vanish identically in $\nu$ only for $\xi=0$, whence our claim by (17).

(B) implies (C) for $P$. First we show that, for a suitable choice of the basis $\left(Y_{j}\right)$ of $a$, all $\xi_{j}$ are proportional to integers for $\xi \in \Gamma^{*}$. Let $\alpha_{1}, \ldots, \alpha_{p}$ be the simple roots, and $\omega_{1}, \ldots, \omega_{p}$ the fundamental weights, defined by

$$
2\left\langle\omega_{i}, \alpha_{j}\right\rangle\left(\left\langle\alpha_{j}, \alpha_{j}\right\rangle\right)^{-1}=\delta_{i j},
$$

where $\langle\omega, \alpha\rangle=\omega\left(H_{\alpha}\right)$ is the scalar product defined by $B$ on $a^{*}$. It is known (cf. Dieudonné $\left[3\right.$, p. 121]) that $\Gamma^{*}$ is a sublattice of $\mathbf{Z} \omega_{1}+\cdots+\mathbf{Z} \omega_{p}$ (with 
equality when $K$ is simply connected), and all numbers $2\left\langle\alpha_{i}, \alpha_{j}\right\rangle\left(\left\langle\alpha_{j}, \alpha_{j}\right\rangle\right)^{-1}$ are integers. It follows that the $\left\langle\omega_{i}, \omega_{j}\right\rangle\left\langle\left\langle\omega_{i}, \omega_{i}\right\rangle\right)^{-1}$ are rational numbers and, by Schmidt orthogonalization, there exists an orthogonal basis $\left(\omega_{j}^{\prime}\right)$ of $a^{*}$ such that each $\omega_{i}$ is a linear combination of the $\omega_{j}^{\prime}$ with coefficients in $\mathbf{Z}$; therefore $\Gamma^{*} \subset \mathbf{Z} \omega_{1}^{\prime}+\cdots+\mathbf{Z} \omega_{p}^{\prime}$. Then the dual basis $\left(Y_{j}\right)$ of $\left(\omega_{j}^{\prime} /\left\|\omega_{j}^{\prime}\right\|\right)$ is an orthonormal basis of a such that, for all $\xi \in \Gamma^{*}, 1 \leqslant j<p, \xi_{j}=\xi\left(Y_{j}\right)$ belongs to $\mathbf{Z}\left\|\omega_{j}^{\prime}\right\|$, whence our claim. Let $d_{j}=\left\|\omega_{j}^{\prime}\right\|$. Assuming (B), we write down the dominant terms of $\tau(P)(\xi, \nu)$ as a polynomial of $\nu$ (of degree $m$ ):

$$
\sum a_{k}\left(\nu_{1}^{2}+\cdots+\nu_{p}^{2}\right)^{k}\left(\xi_{1} \nu_{1}+\cdots+\xi_{p} \nu_{p}\right)^{m-2 k}
$$

$(0 \leqslant 2 k \leqslant m)$; the $a_{k}$ are not all zero. The coefficient of $\nu_{j}^{m}$ in this expression is $\sum a_{k} \xi_{j}^{m-2 k}=q\left(\xi_{j}\right)$ (say), whence

$$
\|\tau(P)(, \cdot)\| \geqslant \sum\left|q\left(\xi_{j}\right)\right| \quad(1 \leqslant j \leqslant p) .
$$

Let $A_{j}$ be the (finite) set of roots of $q$ belonging to $\mathbf{Z} d_{j} .|q|$ is bounded from below by $C>0$ on $\mathbf{Z} d_{j} \backslash A_{j}$, so that $\|\tau(P)(\xi, \cdot)\|>C$ whenever $\xi \in \Gamma^{*}$ and for some $j, \xi_{j} \notin A_{j}$. This inequality is still true when all $\xi_{j} \in A_{j}$, since these are a finite set and $\|\tau(P)(\xi, \cdot)\|$ never vanishes on $\Gamma^{*}$ by assumption. Therefore (C) holds with $k=0$, and this proves the proposition.

5.5. REmarks. The split rank one case is different. When $G=\operatorname{SL}(2, C)$, observe that $Z(G)$ itself is generated by $C$ and $C_{J}$. Indeed, it is known that $Z(G)$ is an algebra of polynomials in two variables (two is the dimension of a Cartan subalgebra of $g$, as a real Lie algebra; cf. Dixmier [4, p. 229]); $C$ and $C_{J}$ are algebraically independent in $Z(G)$, since $\xi^{2}+\nu^{2}+4 \nu$ and $i \xi(\nu+4)$ are, as polynomials in $(\xi, \nu)$ (here $\left.\xi \in \Gamma^{*}=\mathbf{Z}, \nu \in a_{c}^{*}=\mathbf{C}, \rho=2\right)$; furthermore they have minimal degree among nonconstant elements of $Z(G)$, whence our claim. Then (B) and (C) are still equivalent, by the argument above. However, taking

$$
P=k^{2} C+C_{J}^{2}-4 i k C_{J}-k^{4}, \quad k \in \mathbf{Z}, k \neq 0,
$$

shows that (B) is strictly stronger than (A): $\tau(P)(\xi, \cdot)$ vanishes for $\xi=k$, not for $\xi=0$.

Turning to more general cases, when $P$ is not necessarily a polynomial of $C$ and $C_{J}$, one should expect that arithmetic properties of the coefficients make (C) strictly stronger than (B). As regards (A) and (B), we mention that on SL $(3, C)$ there exists $P$ belonging to $Z(G)$ but not to the subalgebra generated by $C$ and $C_{J}$ ( $P$ has order 5), which satisfies (A) and not (B).

\section{REFERENCES}

1. A. Benabdallah, L'opérateur de Casimir de SL(2, R) (to appear).

2. A. Cerezo and F. Rouvière, Solution élémentaire d'un opérateur invariant à gauche sur un groupe de Lie compact, Ann. Sci. Ecole Norm. Sup. 2 (1969), 561-581. 
3. J. Dieudonné, Éléments d'analyse. V, Gauthier-Villars, Paris, 1975.

4. J. Dixmier, Algèbres enveloppantes, Gauthier-Villars, Paris, 1974.

5. R. Gangolli, On the Plancherel formula and the Paley-Wiener theorem for spherical functions on semisimple Lie groups, Ann. of Math. (2) 93 (1971), 150-165.

6. V. Guillemin, The Plancherel formula for the complex semisimple Lie groups, Geometric Asymptotics, Math. Surveys, no. 14, Amer. Math. Soc., Providence, R. I., 1977.

7. S. Helgason, Differential geometry and symmetric spaces, Academic Press, New York, 1962.

8. Fundamental solutions of invariant differential operators on symmetric spaces, Amer. J. Math. 86 (1964), 565-601.

9. symmetric spaces, Math. Ann. 165 (1966), 297-308.

10. in Math. 5 (1970), 1-154.

11. The surjectivity of invariant differential operators on symmetric spaces, Ann. of Math. (2) 98 (1973), 451-479.

12. L. Hörmander, Linear partial differential operators, Springer-Verlag, Berlin, 1963.

13. K. Johnson, Differential equations and an analogue of the Paley-Wiener theorem for linear semisimple Lie groups, Nagoya Math. J. 64 (1976), 17 - 29.

14. B. Kostant, On convexity, the Weyl group, and the Iwasawa decomposition, Ann. Sci. Ėcole Norm. Sup. 6 (1973), 413-455.

15. J. Rauch and D. Wigner, Global solvability of the Casimir operator, Ann. of Math. (2) 103 (1976), 229-236.

16. F. Rouvière, Solutions distributions de l'opérateur de Casimir, C. R. Acad. Sci. Paris 282 (1976), 853-856.

17. F. Treves, Linear partial differential equations with constant coefficients, Gordon and Breach, New York, 1966.

18. N. Wallach, Harmonic analysis on homogeneous spaces, Dekker, New York, 1973.

19. G. Warner, Harmonic analysis on semisimple Lie groups. I, Springer-Verlag, Berlin, 1972.

20. D. Zelobenko, Harmonic analysis of functions on semisimple Lie groups. II, Math. USSRIzvestija 3 (1969), 1183-1217.

Département de Mathématiques, Untversité de Nice, Parc Valrose, 06034 Nice Cedex, FrancB 